\title{
\begin{tabular}{l|l} 
Mibraries & DSpace@MIT
\end{tabular}
}

\author{
MIT Open Access Articles
}

\section{Single-Polarization Cladding-Pumped Optical Amplifier Without Polarization-Maintaining Gain Fiber}

The MIT Faculty has made this article openly available. Please share how this access benefits you. Your story matters.

Citation: Yarnall, T.M. et al. “Single-Polarization Cladding-Pumped Optical Amplifier Without Polarization-Maintaining Gain Fiber." IEEE Photonics Technology Letters 21.18 (2009): 13261328. (C) Copyright 2009 IEEE

As Published: http://dx.doi.org/10.1109/lpt.2009.2026584

Publisher: Institute of Electrical and Electronics Engineers (IEEE)

Persistent URL: http://hdl.handle.net/1721.1/74255

Version: Final published version: final published article, as it appeared in a journal, conference proceedings, or other formally published context

Terms of Use: Article is made available in accordance with the publisher's policy and may be subject to US copyright law. Please refer to the publisher's site for terms of use. 


\title{
Single-Polarization Cladding-Pumped Optical Amplifier Without Polarization-Maintaining Gain Fiber
}

\author{
Timothy M. Yarnall, Todd G. Ulmer, Member, IEEE, Neal W. Spellmeyer, and David O. Caplan, Member, IEEE
}

\begin{abstract}
We demonstrate a single-polarization claddingpumped Er : Yb optical amplifier using a dual-pass design with a Faraday rotator mirror and nonpolarization-maintaining gain fiber. Over a $25-\mathrm{nm}$ range centered at $1562 \mathrm{~nm}$, the amplifier output exceeds $1 \mathrm{~W}$ and maintains a polarization extinction ratio greater than $22 \mathrm{~dB}$. We investigate enhanced relative intensity noise inherent in the dual-pass configuration and discuss scaling to higher powers.
\end{abstract}

Index Terms-Dual-clad fiber, optical amplifiers, polarizationmaintaining (PM) fiber.

\section{INTRODUCTION}

$\mathbf{P}$ OLARIZATION-MAINTAINING (PM) architectures provide several benefits to free-space lasercom systems: broadband transmit/receive diplexing with a compact layout, elimination of polarization-insensitive coating requirements, spatial tracking based on electrooptic nutation, enhanced receiver sensitivity via single-polarization preamplified receivers, compatability with coherent receivers, compatability with polarization-sensitive superconducting photon counters, and the potential for advanced nonmechanical beam-steering techniques [1]-[7]. PM systems can be implemented either by selecting PM components or by using non-PM single-mode (SM) components in conjunction with an active polarization controller; passive PM components are preferable for their simplicity and the minimization of hardware. However, PM components, and in particular those capable of high-power operation, are available from fewer commercial sources than SM components. Thus, it can be advantageous to implement PM subsystems using non-PM components.

The high-power optical amplifier (HPOA) is a key subsystem of a free-space lasercom transmitter. At a minimum, a traditional single-pass PM HPOA based on $\mathrm{Er}: \mathrm{Yb}$ dual-clad fiber requires PM gain fiber and PM isolators; depending on the implementation, PM pump combiners, PM couplers, and PM taps

Manuscript received May 07, 2009; revised June 15, 2009. First published July 14, 2009; current version published September 04, 2009. This work was supported by the Department of the Air Force under Air Force Contract FA8721-05-C-0002. Opinions, interpretations, conclusions, and recommendations are those of the authors and are not necessarily endorsed by the United States Government.

The authors are with the Massachusetts Institute of Technology Lincoln Laboratory, Lexington, MA 02420 USA (e-mail: timmy@11.mit.edu; ulmer@11.mit. edu; neal@11.mit.edu,doc@11.mit.edu).

Color versions of one or more of the figures in this letter are available online at http://ieeexplore.ieee.org.

Digital Object Identifier 10.1109/LPT.2009.2026584

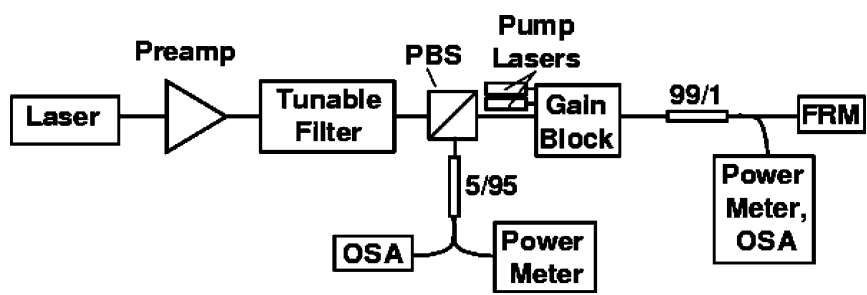

Fig. 1. Dual-pass single-polarization high-power amplifier layout. OSA: optical spectrum analyzer. The pump light enters the gain block travelling in the same direction as light from the PBS.

may also be necessary [8]. Alternatively, a dual-pass configuration based on non-PM core-pumped gain fiber and a Faraday rotator mirror (FRM) has been used to demonstrate PM lasers [9] and low-power PM optical amplifiers [10]-[12]. In this approach, a polarized input signal passes through the gain fiber and undergoes a random polarization rotation. The signal then impinges on the FRM and is both reflected and converted to the orthogonal polarization state. The reflected signal undergoes the same random polarization rotation as on the first pass; however, the sense of the rotation is reversed because the signal is travelling in the opposite direction. Moreover, the forward and backward propagating signals are orthogonally polarized at every point along the fiber.

In this letter, we extend the dual-pass concept to dual-clad HPOAs. We demonstrate a single-polarization amplifier with an output signal power that exceeds $1 \mathrm{~W}$ and a polarization extinction ratio (PER) greater than $22 \mathrm{~dB}$ from 1550 to $1575 \mathrm{~nm}$. We characterize the power, PER, and optical spectra of the HPOA. In addition, we compare the relative intensity noise (RIN) spectra of single-pass and dual-pass configurations that use the same gain fiber. Finally, we discuss scaling to higher powers.

\section{Dual-Pass AmPlifier CONFIGURATION}

The dual-pass HPOA configuration is shown in Fig. 1. The tunable laser and preamp, a commercial low-power PM amplifier, produce a continuous-wave signal power of up to $400 \mathrm{~mW}$. The signal enters the high-power stage via a tunable 1-nm-wide bandpass filter, which suppresses deleterious amplified spontaneous emission (ASE). The filter improves the noise performance but is not absolutely required for operation. A fused-fiber polarization beam splitter (PBS) couples the bandpass filter to the high-power stage, which consists of three parts: a non-PM dual-clad Er: Yb gain fiber, optically pumped by two fiber-coupled 915-nm broad-area diode lasers; an SM 99: 1 coupler, used 


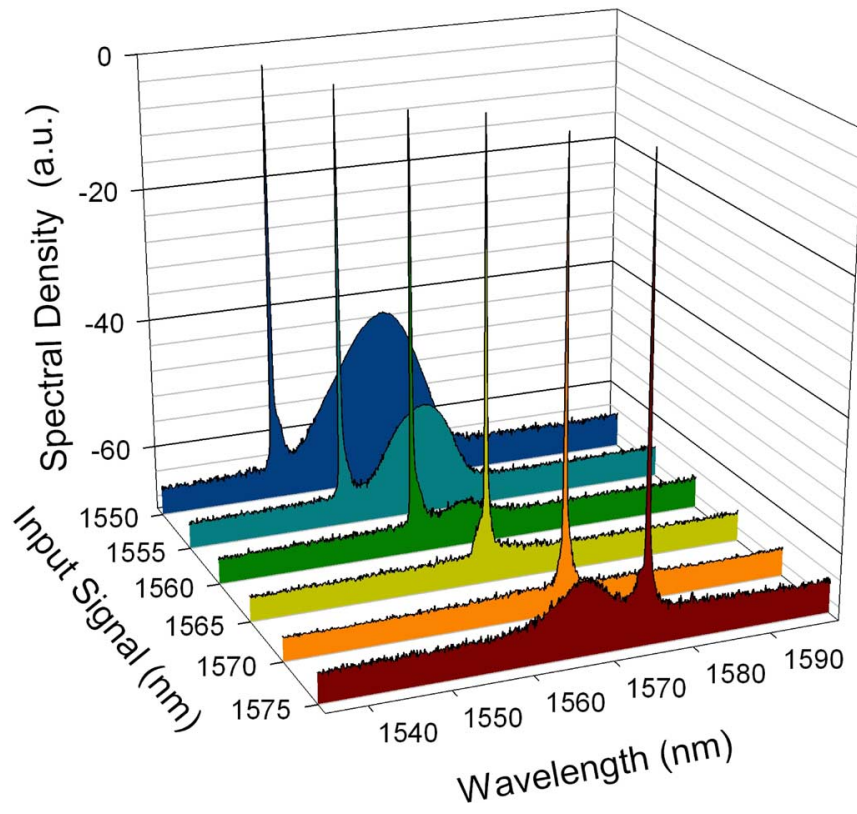

Fig. 2. Output spectra for several input wavelengths $(0.2-\mathrm{nm}$ resolution bandwidth); in each case, the signal output power exceeds $1 \mathrm{~W}$.

for diagnostics; and a fiber-coupled micro-optic FRM. A 95:5 coupler spliced to the output port of the PBS facilitates simultaneous measurement of both the output power and its spectral distribution.

In our particular arrangement, the slow-axis-polarized input signal traverses a low-power PM signal chain before reaching the non-PM high-power gain fiber. The signal then passes through the gain fiber and the monitoring coupler twice, once in the forward direction and, after reflection from the FRM, once in reverse. Next, the signal returns to the PBS which directs it to the output coupler because it is polarized in the fast-axis direction due to the action of the FRM.

\section{AMPLIFIER PERFORMANCE}

The dual-pass HPOA was characterized for power and PER. The output power was measured with a calibrated thermal sensor, corrected for the $4 \%$ Fresnel reflection at the fiber output. The two pump lasers generated up to $8 \mathrm{~W}$ of combined pump power when each was driven with $4.25 \mathrm{~A}$ of current; the peak ASE power for input wavelengths between 1550 and $1575 \mathrm{~nm}$ was greater than $40 \mathrm{~dB}$ below the signal power in a $0.2-\mathrm{nm}$ resolution bandwidth and the output power exceeded $1 \mathrm{~W}$ over the same span. The length of the gain fiber was optimized for a single-pass geometry; improvement in the efficiency is expected if the length were to be tailored for a dual-pass design. At wavelengths shorter than $1550 \mathrm{~nm}$, the amplifier was limited by the gain profile of the particular $\mathrm{Er}: \mathrm{Yb}$ fiber; in Fig. 2, the ASE power, centered at $1565 \mathrm{~nm}$, clearly grows as the input wavelength is tuned from 1570 to $1550 \mathrm{~nm}$. When the input wavelength was tuned below $1550 \mathrm{~nm}$, unstable oscillation began at $1565 \mathrm{~nm}$. The amplifier's gain profile also impacts operation for input wavelengths longer than $1575 \mathrm{~nm}$;

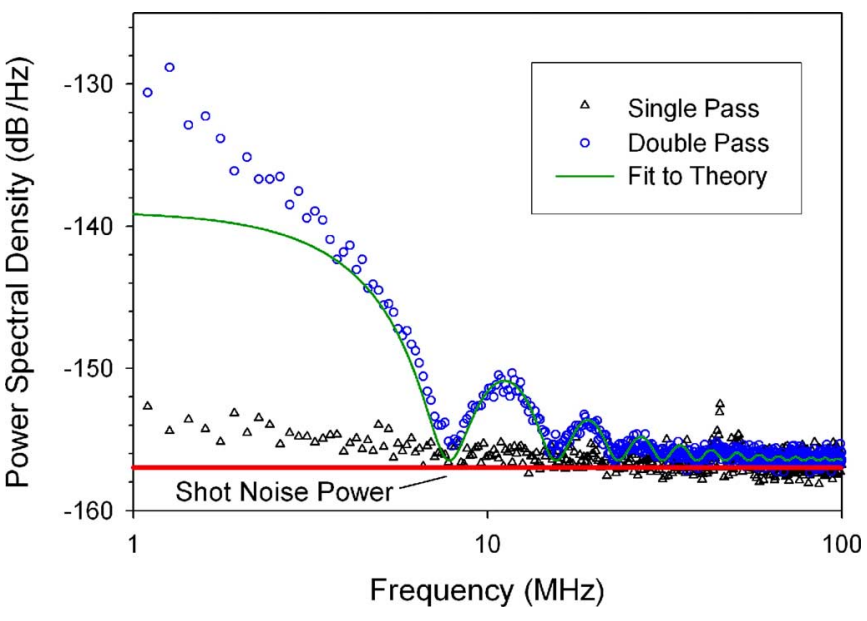

Fig. 3. Measured RIN for a single-pass (black triangles) and double-pass (blue circles) optical amplifier and a fit to theory (green curve).

however, PER degradation at long wavelengths due to the finite bandwidth of the FRM limits the amplifier before the onset of laser action at $1565 \mathrm{~nm}$.

The HPOA's PER was measured via the Poincaré sphere technique [13, Ch. 6] using a commercial polarization analyzer. A PER of greater than $20 \mathrm{~dB}$ is desirable for polarization-diplexed optical communication systems; for inputs from 1550 to $1575 \mathrm{~nm}$, the HPOA output PER exceeded $22 \mathrm{~dB}$. For wavelengths beyond $1575 \mathrm{~nm}$, unwanted cross-coupling between the fast-axis and slow-axis modes resulting from out-of-band operation of both the fused-fiber PBS and the FRM degraded the HPOA's performance: at $1580 \mathrm{~nm}$, the output PER fell below $17 \mathrm{~dB}$. Unlike single-pass PM HPOAs where PER can be degraded by a succession of PM-to-PM splices [14], the PER of the dual-pass design is determined by the input/output PBS and the FRM.

\section{ReLATIVE INTENSITY NOISE}

The dual-pass amplifier configuration has been shown to suffer from higher RIN than single-pass configurations; the increased RIN is attributed to multipass interference caused by finite back-reflections from discrete optical components [15]. To provide a baseline for comparison with the dual-pass configuration, we examined the same gain fiber in a single-pass configuration. We converted the amplifier to a single-pass system by applying a large attenuation via bend loss between the FRM and SM 99: 1 coupler, effectively removing the FRM from the system and providing a $1 \%$ monitor of the single-pass output power.

In Fig. 3, the measured RIN spectra from both single-pass and dual-pass configurations, using the same gain fiber, are compared. The pump current was adjusted to provide the same total gain and output power for each configuration. The single-pass amplifier RIN spectrum was dominated by shot noise and showed modest $1 / f$-type noise. In contrast, the dual-pass amplifier exhibited RIN levels in excess of shot noise 
as well as stronger $1 / f$-type noise. The observed spectrum fit the following equation:

$$
\operatorname{RIN}(f)=\frac{2 R_{p}}{\left(1+R_{p}^{2}\right)} 8 \pi \tau^{2} \Delta \nu_{\mathrm{lw}} \operatorname{sinc}^{2}(\tau f)+\frac{2 h \nu}{P}
$$

where the first term describes phase-noise to intensity-noise conversion via coherent interference between two signals with linewidth $\Delta \nu_{\mathrm{lw}}$, power ratio $R_{p}$, and differential time-delay $\tau$ [13, Appendix A]; the second term describes the shot-noise component of RIN for signal power $P$, where $h$ is Planck's constant and $\nu$ is the center frequency [16]. At low frequencies, the data deviate from the fit because (1) does not include a term for $1 / f$-type noise. The fitted curve corresponded to a signal $93 \mathrm{~dB}$ weaker than the input signal and delayed by $27 \mathrm{~ns}$.

An improvement of approximately $14 \mathrm{~dB}$ in $R_{p}$ would recover the single-pass RIN performance, as would a similar reduction in $\Delta \nu_{\mathrm{lw}}$; in our system, the latter was not a variable. $R_{p}$ could be lessened by reducing either the back-reflection from components that are upstream from the PBS or leakage through the fused-fiber PBS. Optical time-domain reflectometry measurements indicated that a reflection at the FC connectors between the bandpass filter and fused-fiber PBS combined with leakage of the high-power signal through the PBS created the back-reflected signal. Replacing the connector with a fiber-to-fiber splice did not restore the single-pass RIN spectrum; instead, the spectrum shifted, implying the presence of another back-reflection. Repeating the fit provided new values for $\tau$ and $R_{p}$ that implicated the bandpass filter as the source of a back-reflected signal $94 \mathrm{~dB}$ weaker than the primary input. Further reduction of the dual-pass RIN was not attempted.

\section{DISCUSSION}

The 1-W HPOA demonstrated is sufficient to close a preamplified 1-Gb/s differential-phase-shift-keyed link from the moon to geosynchronous orbit, assuming one-foot unobscured apertures and reasonable values for implementation losses and coding gain. The 22-dB PER ensures the amplifier's suitability for polarization-diplexed applications. The increased RIN of the double-pass amplifier makes it less desirable than a single-pass amplifier for analog applications; however, for digital applications, the degraded RIN performance is less of a concern.

The dual-pass configuration is readily scalable to higher powers; our present configuration is limited by the available pump power rather than by damage thresholds. The primary components of concern for damage are the FRM and the PBS. FRMs rated for $2-3 \mathrm{~W}$ are commercially available; they could support HPOA output powers in excess of $10 \mathrm{~W}$. We have selected a fused-fiber PBS to accommodate high output power; however, multi-watt-rated micro-optic PBSs and PM circulators are commercially available and could also be used. An additional benefit of the PM circulator is the possibility of creating a dual-polarization HPOA that can amplify both fast- and slow-axis input signals. The wavelength range of the dual-pass HPOA is limited by the PER of the FRM and the PBS; with different components, the same basic design can be tuned to operate in desired windows across the spectrum supported by the $\mathrm{Er}: \mathrm{Yb}$ gain medium.

\section{CONCLUSION}

We have demonstrated a 1-W single-polarization HPOA with a PER greater than $22 \mathrm{~dB}$ over $1550-1575 \mathrm{~nm}$. The design takes advantage of the high-power capabilities of a cladding-pumped $\mathrm{Er}$ : Yb gain fiber and uses a double-pass configuration with an FRM to achieve single-polarization operation without PM gain fiber. This configuration is readily scalable to powers in excess of $10 \mathrm{~W}$.

\section{REFERENCES}

[1] T. E. Knibbe and E. A. Swanson, "Spatial tracking using an electrooptic nutator and a single-mode optical fiber," Proc. SPIE, vol. 1635, pp. 309-317, 1992.

[2] D. P. Resler, D. S. Hobbs, R. C. Sharp, L. J. Friedman, and T. A. Dorschner, "High-efficiency liquid-crystal optical phased-array beam steering," Opt. Lett., vol. 21, no. 9, pp. 689-691, 1996.

[3] S. Alexander, Optical Communication Receiver Design, ser. SPIE Tutorial Texts in Optical Engineering. Bellingham, WA: SPIE Optical Engineering Press, 1997, vol. TT22, ch. 7.

[4] M. R. Garcia-Talavera, S. Chueca, A. Alonso, T. Viera, and Z. Sodnik, Analysis of the Preliminary Optical Links Between Artemis and the Optical Ground Station, J. C. Ricklin and D. G. Voelz, Eds. Bellingham, WA: SPIE, 2002, vol. 4821, pp. 33-43.

[5] R. Lange and B. Smutny, "Highly-coherent optical terminal design status and outlook," in 2005 Dig. LEOS Summer Topical Meetings, Jul. 2005, pp. 55-57.

[6] A. Biswas, M. W. Wright, J. Kovalik, and S. Piazzolla, Uplink Beacon Laser for Mars Laser Communication Demonstration (MLCD), G. S. Mecherle, Ed. : SPIE, 2005, vol. 5712, pp. 93-100.

[7] V. Anant, A. J. Kerman, E. A. Dauler, J. K. W. Yang, K. M. Rosfjord, and K. K. Berggren, "Optical properties of superconducting nanowire single-photon detectors," Opt. Express, vol. 16, no. 14, pp. 10750-10761, 2008.

[8] P. Wysocki, T. Wood, A. Grant, D. Holcomb, K.-W. Chang, M. Santo, L. Braun, and G. Johnson, "High reliability $49 \mathrm{~dB}$ gain, $13 \mathrm{~W}$ PM fiber amplifier at $1550 \mathrm{~nm}$ with $30 \mathrm{~dB}$ PER and record efficiency," in Optical Fiber Communication Conf. Exposition and The National Fiber Optic Engineers Conf., 2006, pp. PDP17-PDP17.

[9] T. F. Carruthers and I. N. Duling, III, "10-GHz, 1.3-ps erbium fiber laser employing soliton pulse shortening," Opt. Lett., vol. 21, no. 23, pp. 1927-1929, 1996.

[10] I. N. Duling, III and R. D. Esman, "Single-polarisation fibre amplifier," Electron. Lett., vol. 28, no. 12, pp. 1126-1128, Jun. 1992.

[11] F. Hakimi, E. Kintzer, and R. Bondurant, "High-power single-polarization EDFA with wavelength-multiplexed pumps," in Tech. Dig. Lasers and Electro-Optics, 1998 (CLEO 98), May 1998, Summaries of Papers Presented at the Conf., pp. 287-288.

[12] D. Caplan and F. Hakimi, "A high-power high-gain single-polarization EDFA," in Conf. Lasers and Electro-Optics, 2000 (CLEO 2000), 2000, pp. 283-284.

[13] Fiber Optic Test and Measurement, ser. Hewlett-Packard Professional Books, D. Derickson, Ed. Upper Saddle River, NJ: Prentice-Hall PTR, 1998.

[14] M. Monerie, "Polarization-maintaining single-mode fiber cables: Influence of joins," Appl. Opt., vol. 20, no. 14, pp. 2400-2406, 1981.

[15] P. Biernacki, L. T. Nichols, and P. J. Matthews, "Noise effects in polarization maintaining optical amplifiers," in Proc. Optical Fiber Communication Conf., 2000, p. WM3.

[16] S. Alexander, Optical Communication Receiver Design, ser. SPIE Tutorial Texts in Optical Engineering. Bellingham, WA: SPIE Optical Engineering Press, 1997, vol. TT22, ch. 5. 